\title{
DISTRIBUCIÓN DE LOS POLIQUETOS DE FONDOS BLANDOS ENDÉMICOS DE FIORDOS Y CANALES CHILENOS.
}

\author{
DISTRIBUTION OF ENDEMIC SOFT BOTTOM POLYCHAETES \\ FROM CHILEAN FJORDS AND CHANNELS.
}

Américo Montiel ${ }^{1}$ \& Nicolás Rozbaczylo²

\begin{abstract}
Por su geografía y características los fiordos y canales Fuego-Patagónicos conforman un área claramente distintiva de la costa chilena que se extiende desde los $42^{\circ}$ hasta los $56^{\circ}$ de latitud sur. El perímetro de las islas y penínsulas que la componen, posee alrededor de $84.000 \mathrm{~km}$ de línea de costa, mientras que en línea recta sólo se extiende por alrededor de $1.000 \mathrm{~km}$. Esta zona es considerada una de las áreas estuarinas más extensas del planeta $y$, al mismo tiempo, por su aislamiento y problemas de conectividad, una de las áreas menos conocida en Chile (Silva \& Palma 2006).

En el contexto de la zoogeografía marina, el área de los fiordos y canales en Chile ha sido objeto de varias clasificaciones. Forbes (1854), basado en la distribución de especies de peces, moluscos y equinodermos, reconoce dos divisiones en esta área, denominadas "Araucana" y "Fueguina". Ekman (1935), utilizando un conjunto más amplio de especies de invertebrados marinos, denominó toda el área como "Sudamérica Antiboreal". Balech (1954) fue el primero en proponer un esquema zoogeográfico para la llamada región de Magallanes, subdividiéndola en 5 distritos: "Valdiviano" y "Chiloensis", en la costa Pacífica, "Santacruceño" y "Chubutiano" en la costa Atlántica, y un quinto distrito, "Fueguino",
\end{abstract}

el cual conecta ambas costas de la isla grande de Tierra del Fuego.

Luego de numerosas revisiones y esquemas zoogeográficos propuestos en la literatura (e.g. Hedgpeth 1979, Viviani 1979, Brattström \& Johanssen 1983, Stuardo \& Valdovinos 1992, Briggs 1995, Lancellotti \& Vásquez 1999, 2000 y Fernández et al. 2000, entre otros), aún está en discusión la extensión de la región de Magallanes hacia la costa Atlántica como una sola unidad biogeográfica (Camus 2001). Sólo en las últimas décadas, los poliquetos de esta área han sido incluidos en los análisis biogeográficos (Lancellotti \& Vásquez 1999, Glasby \& Álvarez 1999, Fernández et al. 2000). Montiel et al. (2005), basados en la distribución geográfica de los poliquetos, y siguiendo la nomenclatura biogeográfica de Longhurst (1998), determinaron que los fiordos y canales conforman una sola unidad biogeográfica que denominaron "Provincia de Cabo de Hornos", en relación con la corriente marina, y que es diferente tanto de la que se encuentra al norte de ella, "Provincia de Humboldt", como la del lado Atlántico, "Provincia de Falkland". Es así como el esquema zoogeográfico de la región de fiordos y canales no ha sido permanente en el tiempo y sus características aún son poco descriptivas.

1 Laboratorio de Hidrobiología, Instituto de la Patagonia, Universidad de Magallanes, Punta Arenas, Chile. americo.montiel@umag.cl

2 Departamento de Ecología, Facultad de Ciencias Biológicas, Pontificia Universidad Católica de Chile. nrozbaczylo@bio.puc.cl 
Un criterio utilizado tradicionalmente para establecer áreas biogeográficas ha sido el porcentaje de especies endémicas que están presentes en esas áreas. Por lo tanto, el porcentaje de endemismo determinado para un área, varía de acuerdo con la escala espacial y el nivel taxonómico elegido (Lomolino et al. 2005). Por ejemplo, en la región biogeográfica de Magallanes se han registrado 29 especies endémicas de crustáceos decápodos (Gorny 1999), 16 de cumáceos (Mühlenhardt-Siegel 1999) y 62 de isópodos (Brandt 1999); mientras que de moluscos gastrópodos, bivalvos, nudibranquios, aplacóforos y escafópodos hay 25, 22, 11, 9 y 3 especies endémicas respectivamente (Brandt et al. 1999, Schrödl 1999).

En cuanto a los poliquetos endémicos de la región de Magallanes, Ehlers en el año 1901 registró 115 especies; Knox (1977) consideró 223 especies registradas a menos de $500 \mathrm{~m}$ de profundidad, señalando un $16 \%$ de poliquetos endémicos para esta región. Recientemente, Moreno et al. (2006), analizando la distribución de los poliquetos a lo largo de la costa chilena, reconocieron dos áreas de endemismo, una entre las latitudes $18^{\circ}$ y $38^{\circ} \mathrm{S}$ y otra entre $39^{\circ}$ y $56^{\circ} \mathrm{S}$; con dos máximos, uno entre $36^{\circ}$ y $38^{\circ} \mathrm{S}$ y otro entre $39^{\circ}$ y $41^{\circ} \mathrm{S}$. Estos valores son coincidentes con las áreas donde se ha obtenido la mayor cantidad de información sobre poliquetos de la costa de Chile (Montiel 2005).

En este trabajo damos a conocer las especies endémicas de poliquetos bentónicos de fondos blandos y su distribución en el área de los fiordos y canales Fuego-Patagónicos de Chile.

\section{Base de datos}

La información para este trabajo proviene tanto de los resultados obtenidos en el área de estudio por expediciones científicas, mayoritariamente extranjeras, entre los años 1885 y 1999, como de los resultados de nuestras propias investigaciones, algunos de los cuales no habían sido incluidos en análisis zoogeográficos previos (Rozbaczylo et al. 2005, Rozbaczylo et al. 2006a, Rozbaczylo et al. 2006b, Montiel et al. 2007). Se consideró a todas las especies de poliquetos registradas en los fondos blandos de los fiordos y canales Fuego-Patagónicos que cuentan con información geo-referenciada (latitud y longitud) y profundidad. La información analizada corresponde a 416 muestras obtenidas en 272 estaciones por 16 expediciones científicas (Tabla 1). La condición de especie endémica se estableció para todas aquellas cuyas distribuciones geográficas estuvieran restringidas a fondos blandos de fiordos

TABLA 1. Lista de las campañas científicas realizadas en el área de Magallanes en que se ha obtenido poliquetos y el número de estaciones consideradas en este estudio. En paréntesis el número de muestras obtenidas en cada estación oceanográfica.

\begin{tabular}{|c|c|c|c|}
\hline Campaña & Año & Número de estaciones & Fuente \\
\hline HMS Challenger Expedition & $1873-1878$ & 6 & McIntosh 1885 \\
\hline Swedish Antarctic Expedition & $1901-1904$ & 5 & Hartman 1953 \\
\hline Discovery Investigation & 1926-1927, 1931-1933 & 3 & Monro 1930, 1936 \\
\hline Lund University Chile Expedition & $1948-1949$ & 95 & Wesenberg-Lund 1962 \\
\hline Expedición Mar Chile I & 1960 & 6 & Hartmann-Schröder 1965 \\
\hline USNS Eltanin Expedition & $1962-1966$ & 6 & Hartman 1967, Blake 1983 \\
\hline Italian Oceanographic expedition & 1991 & 16 & Gambi et al. 1999 \\
\hline Joint Magellan Campaign & 1994 & $20(128)$ & Montiel et al. 2005 \\
\hline Joint Magellan Campaign & 1994 & 43 & Montiel et al. 2007 \\
\hline Expedition ANT XIII/4 & 1996 & $4(20)$ & Montiel et al. 2005 \\
\hline Expedición CIMAR Fiordos 2 & 1996 & 19 & Montiel et al. 2004 \\
\hline Expedición CIMAR Fiordos 2 & 1996 & $18(37)$ & Montiel 2005 \\
\hline Universidad de Magallanes & 1999-2001 & 3 & Ríos et al. 2003 \\
\hline PUCK Expedition & 2001 & 6 & Palma et al. 2005 \\
\hline Expedición CIMAR 7 Fiordos & 2001 & 21 & $\begin{array}{l}\text { Rozbaczylo et al. } 2005 \\
\text { Rozbaczylo et al. } 2006 \mathrm{a} \text { y b }\end{array}$ \\
\hline Expedición CIMAR 8 Fiordos & 2002 & 16 & $\begin{array}{l}\text { Rozbaczylo et al. } 2005 \\
\text { Rozbaczylo et al. } 2006 \mathrm{a} \mathrm{y} \mathrm{b}\end{array}$ \\
\hline Expedición Fundación Huinay & 2005 & 1 & Montiel et al. (in prep.) $)^{1}$ \\
\hline
\end{tabular}

1 Montiel, A., V. Häusserman, G. Fosterra G \& N. Riff. (in prep.) On rocky reefs polychaete from Chilean fjord and channels. 
y canales entre los $42^{\circ}$ y $56^{\circ} \mathrm{S}$ (Blake 1983, Rozbaczylo 1985, Orensanz 1990, Rozbaczylo et al. 2005, Rozbaczylo et al. 2006, Montiel et al. 2007).

Los mapas de distribución de las especies endémicas fueron realizados con el programa PanMap (http://www.pangaea.de/software/PanMap/). El estatus taxonómico de las especies fue verificado con la base de datos WORMS (World Register of Marine Species; http://www.marinespecies.org/).

\section{Especies endémicas y su distribución}

Se determinó un total de 12 especies de poliquetos endémicos que habitan en los fondos blandos de los fiordos y canales Fuego-Patagónicos chilenos. Estas 12 especies se distribuyen en 10 familias: dos pertenecen a Polynoidae, dos a Terebellidae y cada una de las restantes ocho especies pertenece a una familia diferente (Tabla 2). De las 12 especies, siete figuran registradas en dos oportunidades en la literatura y dos cuentan con tres registros. Las especies recolectadas con mayor frecuencia han sido Aricidea (Allia) pisanoi, en 8 ocasiones, seguida por Harmothoe campoglacialis y Neoleanira magellanica con 6 y 5 recolecciones, respectivamente.

El terebélido Terebellanice laeviseta se distribuye desde la zona intermareal al sublitoral, mientras

TABLA 2. Especies endémicas del área de Magallanes, su localidad tipo, las estaciones en las que fueron encontradas y su respectivo rango de profundidad. Challenger Expedition: CHL; Swedish Antarctic Expedition: SWA; Discovery Investigation: WS; Lund University Chile Expedition: LUCE; Mar Chile I: MCH; USNS Eltanin: ELT; Italian Oceanographic expedition: ITA; Joint Magellan Campaign: VH; Joint Magellan Campaign: VHT; expedition ANT XIII/4: PS; CIMAR Fiordos 2: VG; CIMAR Fiordos 2 VGT; Universidad de Magallanes: UMAG; PUCK: SO; CIMAR-Fiordos 7: C7; CIMAR-Fiordos 8: C8.

\section{FAMILIA PARAONIDAE}

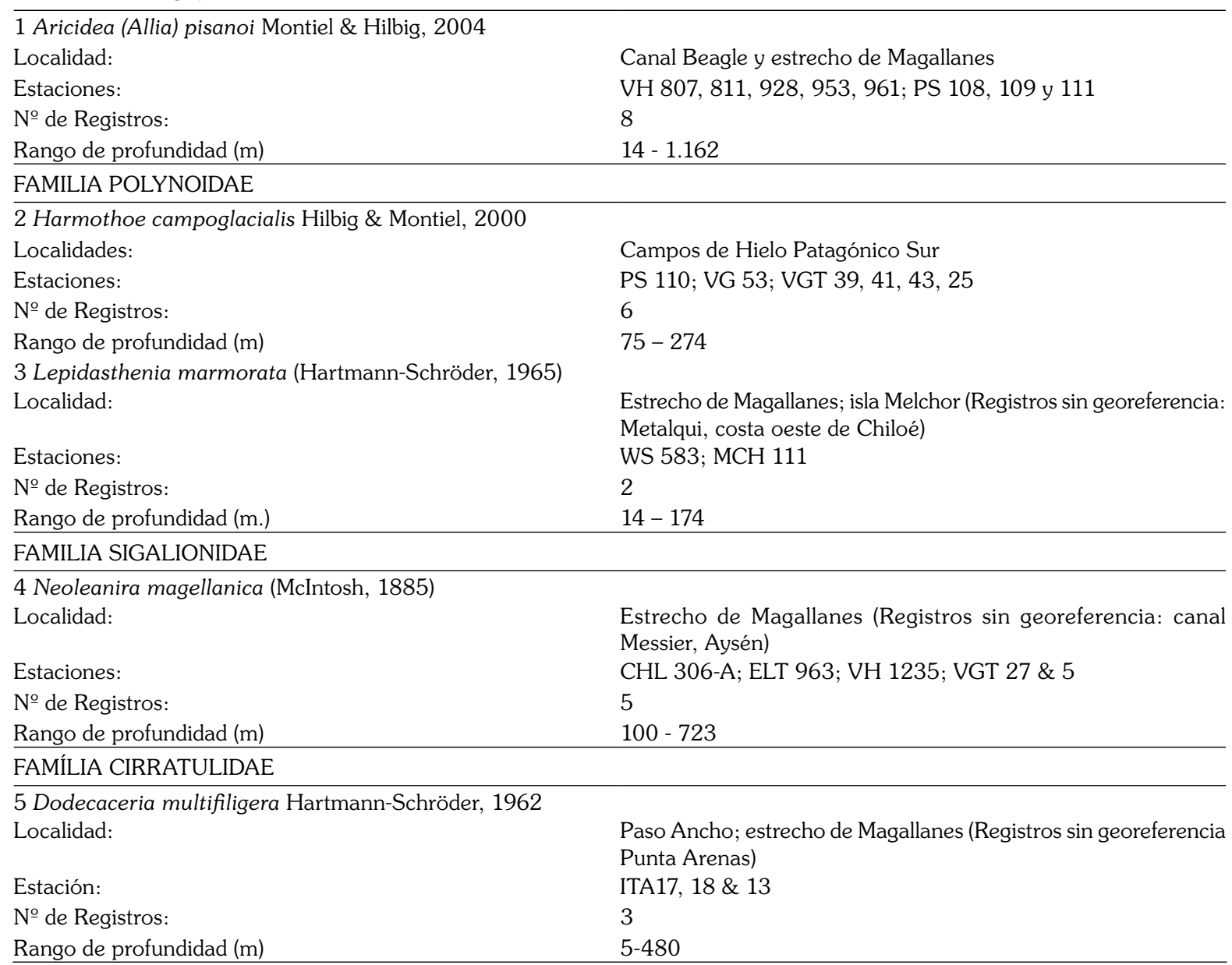




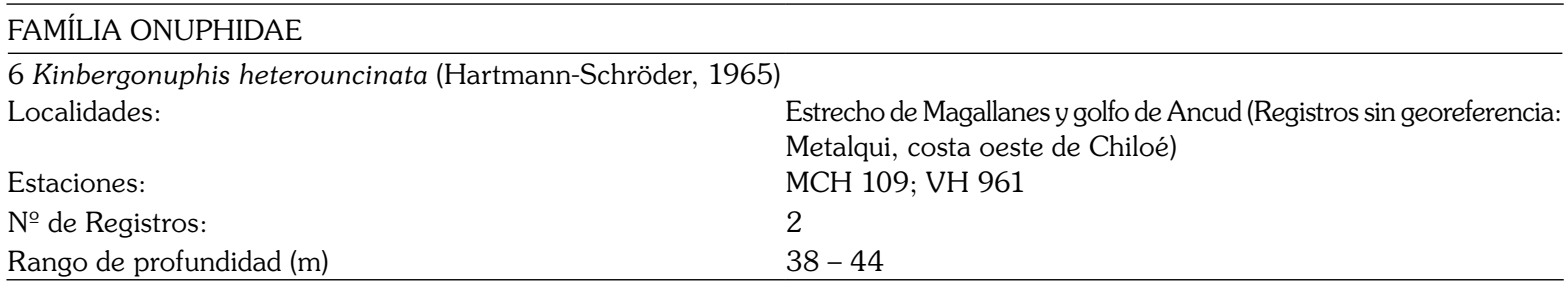

FAMILIA CHAETOPTERIDAE

\begin{tabular}{ll}
\hline 7 Spiochaetopterus patagonicus Kinberg, 1867 & \\
Localidad: & Cabo Vírgenes, estrecho de Magallanes \\
Estación: & HF3 DGA - HF3AIN \\
№ de Registros: & 2 \\
Rango de profundidad (m) & $20-32$ \\
\hline FAMILIA TEREBELLIDAE &
\end{tabular}

\section{Polycirrus multisetigerus Hartmann-Schröder, 1962}

Localidad:

Estaciones:

№ de Registros:

Rango de profundidad (m)

9 Terebellanice laeviseta Hartmann-Schröder, 1962

Localidad:

Estaciones:

№ de Registros:

Rango de profundidad (m)

FAMÍLIA EUPHROSINIDAE
Isla James (Pto. Lagunas),

$\mathrm{MCH} 111$

2

174

Puerto Montt e isla Melchor

C7 $29 \& 21^{a}$

3

intermareal-166

\begin{tabular}{ll}
\hline 10 Euphrosine setosissima Ehlers, 1900 & \\
Localidades: & Estrecho de Magallanes, Puerto Harris (Registros sin georeferencia: \\
& 15 brazas, fondo de rodados con conchillas vacías) \\
Estaciones: & ITA 16 \\
№ de Registros: & 2 \\
Rango de profundidad (m) & $15-115$ \\
\hline
\end{tabular}

FAMILIA SPIONIDAE

11 Dipolydora magellanica (Blake, 1983)

Localidades:

Estrecho de Magallanes

Estaciones:

ELT 960 - VH 961

№ de Registros:

2

Rango de profundidad (m)

$38-64$

FAMILIA SERPULIDAE

12 Hyalopomatus nigropileatus (Ehlers, 1900)

Localidades:

NE del cabo de Hornos (Registros sin georeferencia: estrecho de Magallanes, bahía Harris)

Estaciones:

ELT 219

№ de Registros:

2

Rango de profundidad (m)

$15-115$

que las 11 especies restantes se distribuyen sólo en el sublitoral (Tabla 2); entre ellas, el paraonido Aricidea (Allia) pisanoi presenta el mayor rango de distribución batimétrica, desde 14 a 1.162 m de profundidad. En cuanto a la distribución dentro del

área de fiordos y canales, dos especies (Polycirrus multisetigerus y Terebellanice laeviseta) han sido registradas entre los $42^{\circ}$ y $46^{\circ} \mathrm{S}$ (Fig. 1B); ocho especies se distribuyen entre los $53^{\circ}$ y $56^{\circ} \mathrm{S}$. Entre estas últimas, Neoleanaria magellanica y Harmothoe 

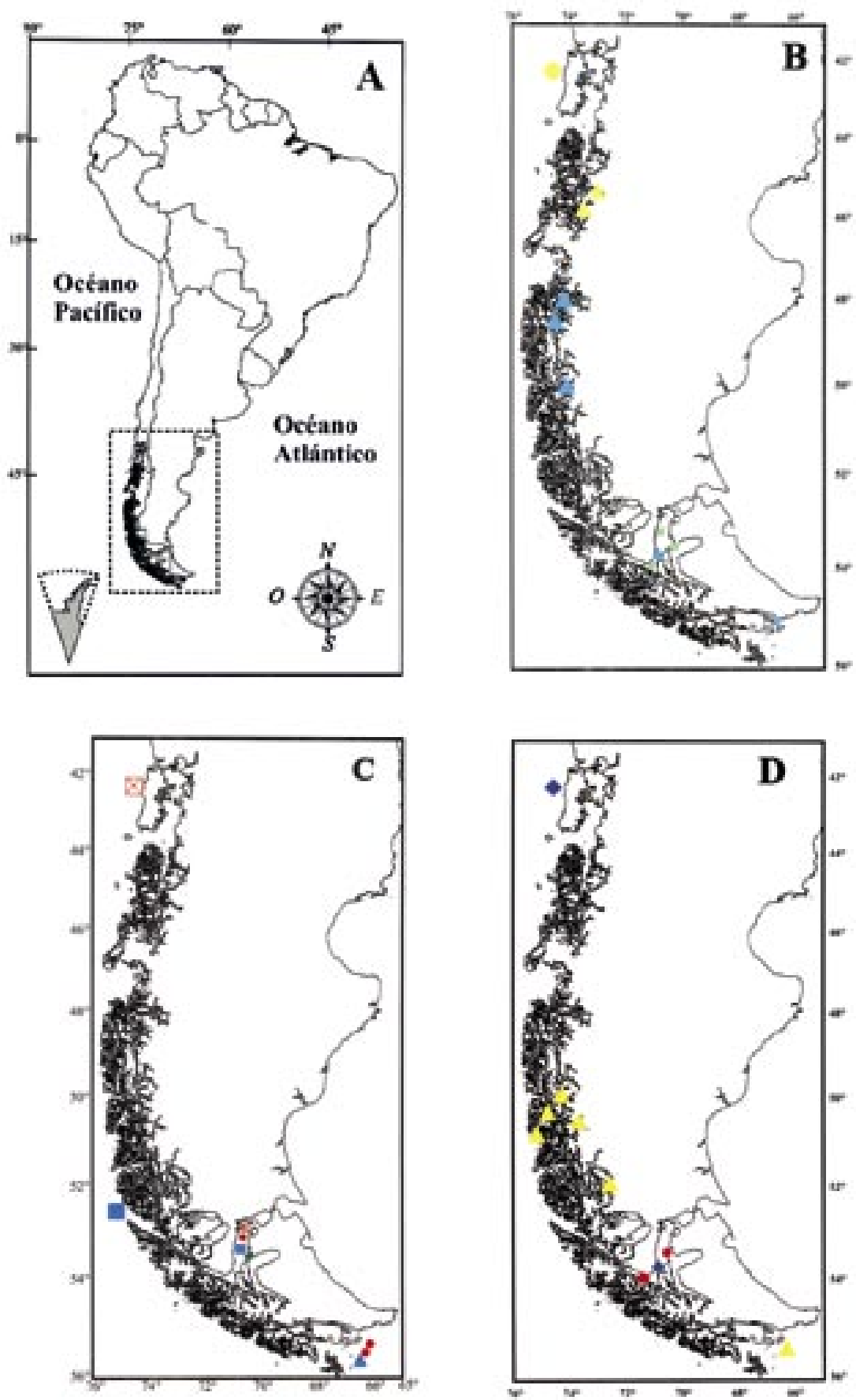

Fig. 1. (A) Área de fiordos y canales Fuego-patagónicos en el territorio chileno. (B) Distribución geográfica de las especies de poliquetos endémicos del área de Magallanes. En Circulo amarillo: Polycirrus multisetigerus; Triángulo amarillo: Terebellanice laeviseta; Triángulo celeste: Neoleanira magellanica; Cuadrado verde: Dodecaceria multifiligera. (C) Cuadrado cruz: Kinbergonuphis heterouncinata; Círculo rojo; Arcidea (Allia) pisanoi; Cuadrado celeste: Dipolydora magellanica; Rombo verde: Euphrosine setosissima; Triángulo azul: Hyalopomatus nigropileatus. (D) Rombo violeta: Lepidasthenia marmorata; Triángulo amarillo: Harmothoe campoglacialis. Círculo rojo: Spiochaetopterus patagonicus. 
campoglacialis muestran distribución más o menos continua entre los canales adyacentes a Campos de Hielo Sur y el canal Beagle (Fig. 1C y 1D); en tanto que Kinbergonuphis heterouncinata y Lepidasthenia marmorata muestran una distribución más bien disjunta ubicándose en los extremos de la región de fiordos y canales (Fig. $1 \mathrm{~B}$ y 1D).

\section{Porcentaje de endemismo}

Varios autores han abordado la definición zoogeográfica del área de los fiordos y canales Fuego-Patagónicos Chilenos, considerándola como región, provincia o subdividiéndola en distritos tomando en consideración uno o varios grupos de organismos marinos (Balech 1954, Hedgpeth 1979, Viviani 1979, Venegas \& Sielfeld 1979, Brattström \& Johanssen 1983, Stuardo \& Valdovinos 1992, Lancellotti \& Vásquez 1999). Sin embargo, debido a la limitada información oceanográfica-física y al inventario taxonómico incompleto, el esquema zoogeográfico del área de fiordos y canales ha sido variable en el tiempo. En este contexto, en 1994, con el desarrollo de la campaña conjunta Chileno-Alemana-Italiana, a bordo del R/V "Victor Hensen" (Arntz \& Gorny 1996) y a partir de 1995, con el desarrollo del programa CIMAR Fiordos organizado por el Comité Oceanográfico Nacional (CONA), a bordo del AGOR "Vidal Gormaz" (Silva \& Palma 2007) se ha logrado un importante y sostenido avance en la completación del inventario faunístico y en la prospección de las áreas hasta entonces no investigadas al interior de los fiordos y canales. A pesar de estos avances, en lo que respecta a poliquetos, en el área de fiordos y canales existen aún grandes desigualdades en términos del esfuerzo de muestreo, generando áreas bien estudiadas y, en otros casos, áreas con ningún tipo de información (Montiel 2005).

Las 12 especies de poliquetos consideradas como endémicas en el área de los fiordos y canales Fuego- Patagónicos chileno, representan sólo el 4\% de la poliquetofauna total registrada en el área y que alcanza a 305 especies. Si consideramos a la región de Magallanes con una costa del Pacífico y una del Atlántico, el porcentaje de endemismo aumentaría a sólo un 7 \% (Montiel et al. 2005). Por lo tanto, el porcentaje de endemismo de poliquetos es el más bajo determinado para la región de Magallanes, contrastando con los porcentajes de endemismo señalados para otros grupos de invertebrados bentónicos, como isópodos (50\%), y varios taxones de moluscos como gastrópodos (48\%), bivalvos (42\%), nudibranquios (31\%), aplacóforos (100\%) y escafópodos (60\%) (Brandt et al. 1999, Schrödl 1999).

Las especies no endémicas de poliquetos presentes en esta región muestran diversos patrones de distribución geográfica, pudiendo agruparse de la siguiente manera: un 35\% distribuida en torno al cono sur de Sudamérica; $32 \%$ desde la costa de Chile continental hasta áreas del océano austral (Subantártica y Antártica); 4\% en ambas costas del cono sur de Sudamérica hasta áreas del océano austral; un $12 \%$ de las especies son consideradas cosmopolitas, y el $13 \%$ restante muestra una distribución disjunta (Montiel et al. 2005).

Una característica de la poliquetofauna del área de los fiordos y canales, es la presencia de un alto porcentaje (32\%) de especies con un amplio rango de distribución geográfica, que se extiende hasta áreas subantárticas y antárticas. Esta característica también se ha observado en otros grupos de invertebrados bentónicos como crustáceos decápodos y moluscos (Gorny 1999, Linse et al. 1999). Montiel et al. (2005), sugieren que el bajo porcentaje de poliquetos endémicos que se observa en el área de los fiordos y canales Fuego-Patagónicos puede ser el reflejo de la historia glaciológica de esta área y también de la apertura del estrecho de Magallanes. Sin embargo, el rol de estos procesos históricos, que sin duda modelan el patrón biogeográfico actual, es escasamente entendido. El bajo esfuerzo de muestreo desplegado hasta ahora en el área de los fiordos y canales se refleja también en el bajo número de registros de las especies endémicas, los que varían entre 1 y 8 registros por especie.

Los valores de endemismo calculados para una determinada área de estudio, en un determinado momento, pueden variar con el esfuerzo de muestreo. A medida que se realizan más prospecciones en dicha área, él número de especies encontradas puede aumentar y, por consiguiente, variar el porcentaje de endemismo. Así, el bajo porcentaje de poliquetos endémicos mostrado actualmente en el área fiordos y canales Fueguino -Patagónicos de la región de Magallanes deberá ser confirmado más adelante a la luz de nuevas investigaciones. 


\section{AGRADECIMIENTOS}

Este trabajo fue realizado con el financiamiento del Programa Interno PH-F3-01LH-06-07 de la Dirección de Investigación, Universidad de Magallanes. Agradecimientos a la Dr. Vreni Häussermann y a Dr. (c) Günter Försterra por el material de poliquetos aportados de la expedición de la Fundación Huinay. También se agradece al Dr. David Rumsey por su valiosa colaboración.

\section{LITERATURA CITADA}

Arntz, W.E. \& M. Gorny 1996. Cruise report of the Joint Chilean-German-Italian "Victor Hensen" Campaign in 1994. Reports on Polar and Marine Research 190: 1-113

Balech, E. 1954. Divisiones zoogeográficas del litoral sudamericano. Revista Biología Marina 4: $184-195$

Blake, J.A. 1983. Polychaetes of the family Spionidae from South America, Antarctica and adjacent seas and islands. Biology of the Antarctic Seas XIV. Antarctic Research Series 39 (3): 205-288.

Brandt, A. 1999. On the origin and evolution of antarctic Peracarida (Crustacea, Malacostraca). In W.E. Arntz and C. Ríos (Eds.) MagellanAntarctic: Ecosystems that drifted apart. Scientia Marina 63 (Suppl.1): 261-274

Brandt, A., K. Linse \& U. Mühlenhardt-Siegel 1999. Biogeography of Crustacea and Mollusca of the Subantarctic and Antarctic regions. In W.E. Arntz and C. Ríos (Eds.) Magellan-Antarctic: Ecosystems that drifted apart. Scientia Marina 63 (Suppl.1): 383-389

Brattström, H. \& A. Johanssen 1983. Ecological and regional zoogeography of the marine benthic fauna of Chile. Sarsia 68: 289-339

Briggs, J.C. 1995. Global biogeography. Elsevier, Amsterdam, The Netherlands. $452 \mathrm{pp}$.

Camus, P. 2001. Biogeografía marina de Chile continental. Revista Chilena de Historia Natural 74: 587-617

Ehlers, E. 1901. Die Polychaeten des magellanischen und chilenischen Strandes. Ein faunistischer Versuch. Festschrift zur Feier des Hundertfünfzigjährigen Bestehens der Königlichen Gesellschaft der Wissenschaften zu Göttin- gen. (Abh. Math.-Phys. K.), Berlin, 232 pp., 25 pls.

Ekman, S. 1935. Tiergeographie des Meeres. Leipzig: Akad. Verl.-Ges.

Fernández, M., E. Jaramillo, P. Marquet, C. Moreno, S. Navarrete, P. Ojeda, C. Valdovinos \& J. Vásquez 2000. Diversidad, dinámica y biogeografía del ecosistema costero bentónico de Chile: revisión y bases para la conservación marina. Revista Chilena de Historia Natural 73 (4): 797-830

Forbes, E. 1854. Zoological geography. The distribution of marine life. In Johnston A. K. (Ed) "The physical atlas of natural phenomena". Engraved \& printed in colours by W. \& A.K. Johnston, Edinr. William Blackwood \& Sons, Edinburgh \& London: 98-102. Plate 31.

Gambi, M. \& S. Mariani 1999. Polychaetes of the soft bottoms of the Strait of Magellan collected during the Italian oceanographic cruise in february-march 1991. In W.E. Arntz and C. Ríos (Eds.) Magellan-Antarctic: Ecosystems that drifted apart. Scientia Marina 63 (suppl. 1): $233-242$

Glasby, C.H. \& B. Álvarez 1999. Distribution patterns and biogeographic analysis of Austral polychaeta (Annelida). Journal of Biogeography 26: 507-533.

Gorny, M. 1999. On the biogeography and ecology of the Southern Ocean decapod fauna. In W.E. Arntz and C. Ríos (Eds.) Magellan-Antarctic: Ecosystems that drifted apart. Scientia Marina 63(Suppl.1): 367-382

Hartman, O. 1953. Non-pelagic polychaeta of the Swedish Antarctic Expedition 1901-1903. Further Zoological Results of the Swedish Antarctic Expedition 1901-1903, 4 (11): $1-83$.

Hartman, O. 1967. Polychaetous annelids collected by the USNS Eltanin and Staten Island cruises, chiefly from Antarctic Seas. Allan Hancock Monographs in Marine Biology 2: 1-387.

Hartmann-Schröder, G. 1965. Zur Kenntnis des Sublitorals der chilenischen Küste unter besonderer Berücksichtigung der Polychaeten und Ostracoden. Tl. II. Die Polychaeten des Sublitorals. Mitteilungen aus Hamburgischen Zoologischen Museum und Institut 62(suppl.): 59-305. 
Hedgpeth, J.W. 1969. Distribution of selected groups of marine invertebrates in waters south of $35^{\circ}$ S latitude. Antarctic Map Folio Ser, American. Geography Society 11: 1-4

Knox, G.A. 1977. The Antarctic polychaete fauna: its characteristics, distribution patterns, and evolution. In: G.A. Llano (ed.), Adaptation within Antarctic Ecosystem, Proc. III SCAR Symp. Antarct. Biol. Smithsonian Institution, Washington D. C: 1111-1127

Lancellotti, D.A. \& J.A. Vásquez 1999. Biogeographical patterns of benthic macroinvertebrates in the Southeastern Pacific littoral. Journal of Biogeography 26: 1001-1006.

Lancellotti, D.A. \& J. Vásquez 2000. Zoogeografía de macroinvertebrados bentónicos de la costa de Chile: contribución para la conservación marina. Revista Chilena de Historia Natural 73(1): 99-129.

Longhurst, A. 1998. Ecological Geography of the Sea. Academic Press. 398pp

Lomolino, M. V., B. R. Riddle \& J. H. Brown 2005. Biogeography. $3^{\text {ra }}$ edición Sunderland: Sinauer Associates. $845 \mathrm{pp}$

McIntosh, W. C. 1885. Report on the Annelida Polychaeta colleted by H.M.S. Challenger during the years 1873-1876. In: Report on the Scientific Results of the Voyage of H.M.S. Challenger during the Years 1873-1876 under the Command of Captain George S. Nares, R.N., F.R.S. and the Late Captain Frank Tourle Thomson, R.N., Zoology, 12 (34): 1-554, pls. 1-55, and 1a-39a.

Monro, C. C. A. 1936. Polychaete worms. II. Discovery Reports, 12: 59-198.

Montiel, A., C. Ríos, E. Mutschke \& N. Rozbaczylo 2004. Poliquetos de fiordos y canales adyacentes al Campo de Hielo Patagónico Sur, Chile (Annelida: Polychaeta). Ciencia y Tecnología del Mar 27 (1): 49-67.

Montiel, A., D. Gerdes \& W. Arntz 2005. Distribution patterns of shallow waters polychaetes in the Magellan region: a zoogeographical and ecological synopsis. Scientia Marina, 69 (suppl. 2): 123-133

Montiel, A., C. Ríos \& E. Mutschke 2007. Biodiversidad en la costa noroccidental y sur de Tierra del Fuego. Anales Instituto Patagonia (Chile) 35 (2): 41-52
Montiel, A. 2005. Biodiversity, zoogeography and ecology of polychates from the Magellan region and adjacent areas. Reports on Polar and Marine Research, 505: 1-112

Moreno, R.A., C.E. Hernández, M.M. Rivadeneira, M.A. Vidal \& N. Rozbaczylo 2006. Patterns of endemism in southeastern Pacific benthic polychaetes of the Chilean coast. Journal of Biogeography 33 (4): 750-759

Mühlenhardt-Siegel, U. 1999. On the biogeography of Cumacea (Crustacea, Malacostraca). A comparison between South America, the Subantarctic Islands and Antarctica: present state of the art. In W.E. Arntz and C. Ríos (Eds.) Magellan-Antarctic: Ecosystems that drifted apart. Scientia Marina 63(Suppl.1): 295-302

Orensanz, J. M. 1990. The eunicemorph polychaete annelids from Antarctic and Subantartic seas. With addenda to the Eunicemorpha of Argentina, Chile, New Zealand, Australia, and the southern Indian Ocean. Biology of the Antarctic Seas XXI. Antarctic Research Series 52: 1-183.

Palma, M., E. Quiroga, V.A. Gallardo, W. Arntz, W. Schneider, D. Gerdes \& D. Hebbeln 2005. Macrobenthic animal assemblages of the continental margin of Chile $\left(22^{\circ}\right.$ to $\left.42^{\circ} \mathrm{S}\right)$. Journal of Marine Biological Association of the United Kingdom, 85: 233-245

Ríos, C., E. Mutschke \& E. Morrison 2003. Biodiversidad bentónica sublitoral en el Estrecho de Magallanes, Chile. Revista de Biología Marina y Oceanografía 38 (1): 1-12.

Rozbaczylo, N. 1985. Los anélidos poliquetos de Chile. Índice sinonímico y distribución geográfica de especies. Monografías Biológicas 3: $1-284$.

Rozbaczylo, N., R.A. Moreno \& O. Díaz-Díaz 2005. Poliquetos bentónicos submareales de fondos blandos de la Región de Aysén, Chile: Clado Phyllodocida (Annelida, Polychaeta). Investigaciones Marinas 33 (1): 69-89.

Rozbaczylo, N., R.A. Moreno, O. Díaz-Díaz \& S. Martínez 2006a. Poliquetos bentónicos submareales de fondos blandos de la Región de Aysén, Chile: Clado Terebellida (Annelida: Polychaeta). Ciencia y Tecnología del Mar 29(1): 71-90. 
Rozbaczylo, N., R.A. Moreno \& O. Díaz-Díaz $2006 b$. Poliquetos bentónicos submareales de fondos blandos de la región de Aysén, Chile: Clados Amphinomida, Eunicida, Spionida, Sabellida y Scolecida (Annelida, Polychaeta). Investigaciones Marinas 34 (1): 43-62.

Schrödl, M. 1999. Zoogeographic relationships of Magellan Nudibranchia (Mollusca: Opisthobranchia) with particular reference to species from adjacent regions. In W.E. Arntz and C. Ríos (Eds.) Magellan-Antarctic: Ecosystems that drifted apart. Scientia Marina 63(Suppl.1): 409-416

Silva, N. \& S. Palma 2006. Avances en el conocimiento oceanográfico de las aguas interiores chilenas, Puerto Montt a cabo de Hornos. Comité Oceanográfico Nacional - Pontificia Universidad Católica de Valparaíso, Valparaíso, $162 \mathrm{pp}$.
Stuardo, J. \& C. Valdovinos 1992. Barreras, límites faunísticos y provincias biogeoquímicas en Sudamérica austral. In: V.A. Gallardo, O. Ferretti, H.I. Moyano (eds.), Oceanografia in Antartide: atti seminario internazionale, pp. 443-452. ENEA/Centro EULA, Concepción, Chile

Venegas, C. \& W. Sielfeld 1979. Antecedentes para la determinación de un nuevo distrito zoogeográfico en el litoral exterior de Magallanes. Anales Instituto Patagonia (Chile) 10: 201-208.

Viviani, C. 1979. Ecogeografía del litoral chileno. Studies on the Neotropical Fauna and Environment 14: 65-123

Wesenberg-Lund, E. 1962. Polychaeta Errantia. Reports of the Lund University Chile Expedition 1948-1949. (43). Lunds Universitets Årsskrift. N.F. Avd. 2, 57 (12): 1-139. 
A. MONTIEL \& N. ROZBACZYLO 